\title{
Experimental Study of Bone Marrow Stem Cell Inducing Osteogenesis on SLA Titanium Mesh Surface
}

\author{
Lei SHI ${ }^{1}$, Lan YANG ${ }^{2}$, Rui-min LIU ${ }^{1}$, Feng TAO ${ }^{1}$ and Qiao-rong \\ LIU $^{1}$ \\ ${ }^{1}$ Gansu Provincial Hospital Oral Medical Center, China \\ ${ }^{2}$ Lanzhou University Second Hospital Oral and Maxillofacial Surgery, China
}

Keywords: BMSCs, mMedical titanium mesh, SLA, Inducing osteogenesis.

\begin{abstract}
Objective: Through animal experiments to understand medical titanium mesh in the sandblasting acid corrosion (SLA) before and after processing and surface coverage of BMSCs cell membrane after the effect of inducing osteogenesis, for medical titanium net covering the bone marrow stem cells after surface modification diaphragm local defect reconstruction of maxilla and form a bionic bone. Materials and methods: To observe the SLA titanium mesh surface morphology changes before and after processing; Making experimental rabbit BMSCs and culture cell cell membrane, According to the experimental steps and grouping plan will surface treatment before and after the experiment rabbit implanted with titanium mesh and cell membrane, after six months after feeding titanium mesh and the surface osteogenesis effect was observed and compared. Results: 1) Will be without surface treatment and after surface treatment of medical experimental rabbit implanted with titanium mesh directly, after take out the titanium mesh specimen observation, without surface treatment of the surface of the titanium mesh individual islands area is bone tissue osteogenesis effect is poorer, after surface treatment of titanium mesh is formed relatively complete bone tissue layer, but relatively weak bone tissue layer. 2) To the blanket in the diaphragm of BMSCs cells in medical titanium implant experimental animals, after take out the titanium mesh specimen observation, uncovered the cell membrane of titanium net, no matter whether it is for surface treatment, the surface osteogenesis effect are also covered the cell membrane of titanium mesh is poor.Conclusion: Bone marrow stem cell patch plays a decisive role in the process of titanium mesh inducing osteogenesis. Titanium mesh surface SLA processing only plays an auxiliary role. Therefore, the order from the best to the worst final effect of inducing osteogenesis is shown as follows: SLA processing + cell patch > no SLA processing + cell patch $>$ SLA processing $>$ no SLA processing.
\end{abstract}

\section{Introduction}

Local or overall bone defect of facial bone tissue belongs to more common disease clinically. These patients always suffer from many difficulties in treatment. Current restoration methods generally includes two categories: far-end autogenous bone graft and artificial material restoration[1]. The following problems are caused when autologous bone is used for restoring bone defect: firstly, bone source for repairing bone defect bone is scarce relatively; secondly, the operation is more difficult, it is required that the operators $\mathrm{w}$ should be provided with skilled microsurgical operation technology and rich experience in flap operation; thirdly, it is not convenient to shape autologous bone during operation; fourthly, it is also more difficult to prevent and treat postoperative complications; fifthly, patients have worse subjective 
evaluation on remote bone grafting during the treatment process. In addition, the emergence of some cell-free allograft also brings new method for similar operation in recent years. However, the operation is always ended by tissue rejection clinically; Furthermore, when absorbable biomaterials are adopted for restoration, it is expected that biological materials can induce autologous bone formation and creeping substitution absorbable biomaterials can form new bone eventually. However, absorbing speed of absorbable biomaterials and autologous bone ossification speed always cannot be completely consistent. Early strength of absorbable biomaterials and the plasticity during reconstruction of facial bone cannot well meet operation need. There are still more technical difficulties in practical application. Therefore, medical titanium mesh always acts as priority materials in $2 / 3$ large-area bone defect reconstruction treatment in facial part. However, after medical titaniummesh is implanted into the tissue, since surface does not undergo special treatment, patients still can feel various discomforts due to metal materials as permanent implant material [2]. Therefore, surface of titanium mesh should be processed. Certain amount of thin-layer bone can be formed on titanium mesh surface through various methods after the mesh is implanted for certain time. The titanium mesh can achieve higher bionic effect after restoration and reconstruction [3].

\section{Materials and Methods}

\section{Experimental Animal, Instrument and Reagent}

New Zealand newborn rabbits (Gansu University of Traditional Chinese Medicine Animal Experimental Center), medical titanium mesh with specification of $100 \mathrm{~mm} * 80 \mathrm{~mm} * 1 \mathrm{~mm}$ (Xi'an Zhongbang Biological Materials Co., Ltd.); box sand-blasting machine (Zhongshan Baide Machinery Equipment Co., Ltd.), ultrasonic cleaner (Shanghai Yuejin CQ-500AD), balance (Nanjing Electronic Balance Factory), $\mathrm{CO}_{2}$ cell incubator (Ji'nan Zhuoao Biological Technology Co., Ltd.), YJ-875 super-clean workbench (Suzhou Purification Equipment Factory), inverted microscope and camera system (Olympus CX31), field emission scanning electron microscopy (FESEM,JSM-6701F), centrifugal machine (German HeraeusCryofuge 8000); $\mathrm{Al}_{2} \mathrm{O}_{3}$ particles (Beijing Huanqiu Jinding Technology Co., Ltd), $\mathrm{HCl}$ and $\mathrm{H}_{2} \mathrm{SO}_{4}$ (Shanghai Yuqin Chemical Industry Co., Ltd.), deionized water $(>18.2 \mathrm{M} \Omega \cdot \mathrm{cm})$, amino acid and glucose medium (Dulbecco's Modified Eagle Medium, Gibco), fetal bovine serum (Hangzhou Sijiqing), trypsin (Shanghai Yaxin Biological Preparation Co., Ltd.), vitamin C (Henan Runhong Pharmaceutical Co., Ltd.), dexamethasone (Zhengzhou Zhuofeng Pharmaceutical Co., Ltd.), BALP test reagent box (Shanghai Lanpai Biological Technology Co., Ltd).

\section{SLA Processing of Titanium Mesh}

Two medical titanium meshes are removed, one mesh is not processed, and the other mesh undergoes SLA processing. Firstly, the mesh is fixed in sand-blasting box by prefabricated stent during processing, the nozzle pressure is $0.6 \mathrm{MPa}$, and the nozzle should be vertical to titanium mesh surface as far as possible. The shortest distance between the nozzle and the titanium mesh is about $15 \mathrm{~cm} . \mathrm{Al}_{2} \mathrm{O}_{3}$ with particle size of 200-400 microns is adopted for sandblasting processing of titanium mesh for 30s. The mesh is cleaned respectively by deionized water, anhydrous acetone and ethanol ultrasonically in turn for $5 \mathrm{~min}$ after processing, and then the mesh is dried by blowing. Then, acid etching processing is implemented. Mixed pickling solution is prepared temporarily before acid etching. The preparation proportion is 
$18 \% \mathrm{HCl}$ and $48 \% \mathrm{H}_{2} \mathrm{SO}_{4}$. The titanium mesh after sandblasting processing is submerged in mixture of hydrochloric acid and sulfuric acid, and acid treatment lasts for $15 \mathrm{~min}$. Cleaner is used for ultrasonic cleaning and drying after completion of acid etching completion. After titanium mesh undergoes SLA processing, optical microscope and scanning electron microscopy are applied for respectively observing the changes of the surface morphology as shown in figure 1.

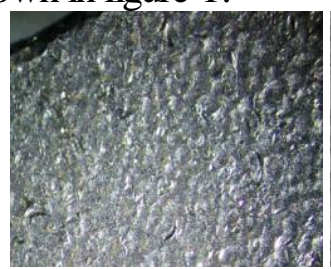

A

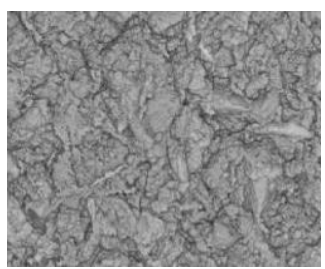

B

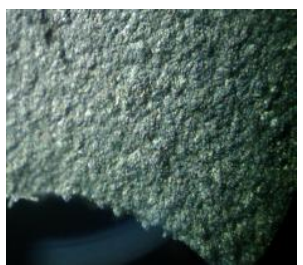

$\mathrm{C}$

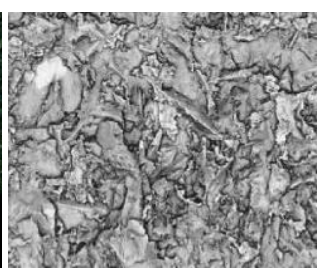

$\mathrm{D}$

Figure 1.The pictures of Titanium mesh surface before and after SLA treatment from optical microscope (x40) and scanning electron microscope (x2000). A. Untreated titanium mesh from optical microscope; B.

Untreated titanium mesh from SEM; C. Treated titanium mesh from optical microscope; D. Treated titanium mesh from

\section{Cell Patch Cultivation}

Firstly, bone marrow tissue is prepared, six New Zealand newborn rabbits born for 14 days are adopted, and fresh red bone marrow is obtained under aseptic operation. When red bone marrow is adopted, the inner wall of $1 \mathrm{ml}$ syringe undergoes heparinization processing in advance. Red bone marrow $0.2 \mathrm{ml} \sim 0.3 \mathrm{ml}$ is taken. Bone marrow mesenchymal stem cells are cultivated by full bone marrow adherence method. Passage cells in the third generation for subsequent experiment are finally obtained. Then, bone marrow mesenchymal stem cell patch is obtained in vitro in the laboratory through simple cell patch technology. After seed cells are obtained, simple cell patch technology is utilized for cultivating cell patch. Main strategies of simple cell patch technology: vitamin $\mathrm{C}$ bone marrow mesenchymal stem cells are added in medium for proliferation and secretion of extracellular matrix. Then, 3D polymer with both seed cells and extracellular matrix can be obtained. Cell sheet thickness changes in the cultivation process are observed. The bottom of culture dish should not be contacted as far as possible during liquid absorption and liquid addition in cell patch cultivation. The integrity of cell patch cannot be guaranteed as far as possible. When the cell folds on the edge of cell patch are observed, it is displayed that the cell patch has been basically formed. The humidity of the cell culture dish should be kept as far as possible during the whole process of cell patch cultivation. Then, softer blunt latex tweezers should be used for clipping cell sheet. Then cell sheet and culture dish are completed separated [4-10]. Cultivation cell patch process is shown in figure 2 . 


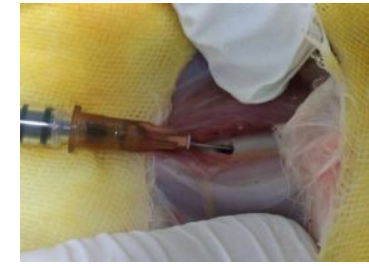

A

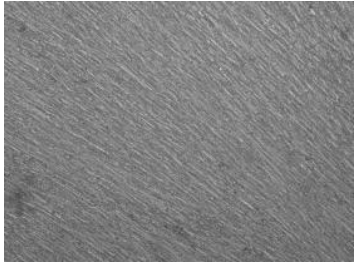

$\mathrm{D}$

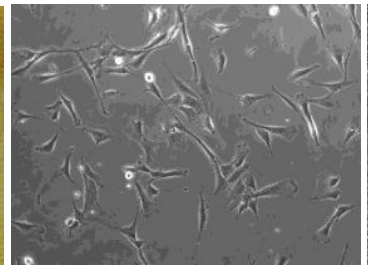

B

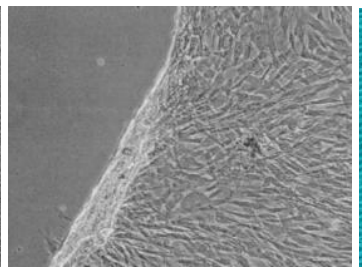

E

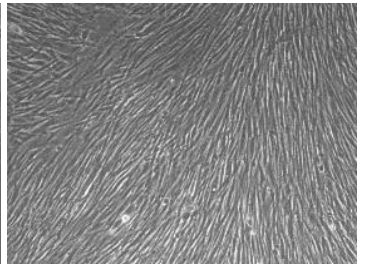

C

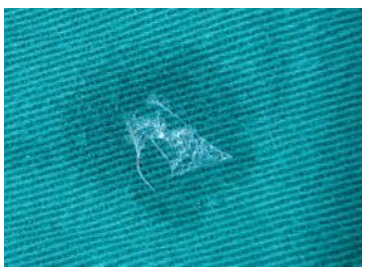

$\mathrm{F}$

Figure 2.The cultivation of the cell membrane process. A. Get red marrow;B. BMSC adherent growth day 1;C. BMSC adherent growth day 3;D. BMSC adherent growth day 7; E. The edge of BMSC cell membrane; F. Can be used in the operation of the cell membrane.

\section{Establishment of Animal Model}

Forehead skin of experimental rabbit is cut under anesthesia state. High speed round burr is cooperated with physiological saline for cooling. Skull bone of about $2 \mathrm{~cm} * 2 \mathrm{~cm}$ is removed in a disrupted mode. Dura mater external operation should be gentle as far as possible in order to prevent abnormal vital signs of experimental animals. Some bones for fixing screws should be reserved on wound edges. Titanium mesh after surface processing and titanium mesh without surface processing should be implanted into bone defect synchronously according to plan. Controls can be formed. Cell patch is covered on titanium mesh surface according to experiment plan aiming at other experiment rabbits. After titanium mesh is implanted, the wound should be flushed. After active bleeding is not discovered according to observation, the skin and subcutaneous tissue should be carefully sewn. After experimental animals wake up completely, the vital signs are observed, the rabbits are returned back to the cage after the vital signs become stable. Gentamicin intramuscular injection is implemented twice a day for seven consecutive days until the wounds are completely healed as shown in figure 3.

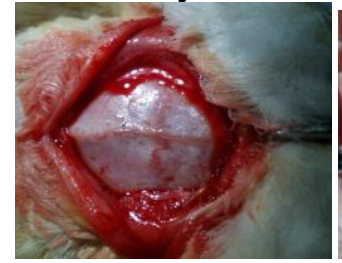

A

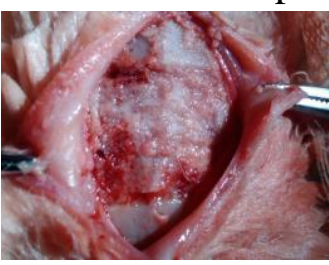

$\mathrm{B}$

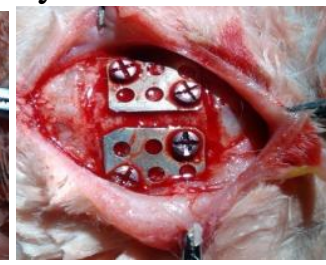

$\mathrm{C}$

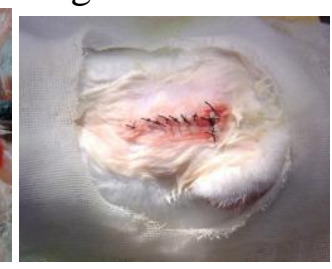

$\mathrm{D}$

Figure 3. The process of establishing animal model. A. Skin incision and skull exposure;B. To remove the skull in about $2 \mathrm{~cm} * 2 \mathrm{~cm} ; \mathrm{C}$. Install the titanium mesh;D. Closing a wound.

\section{Specimen Preparation}

After experimental animals are fed for six consecutive months, skin and periosteum are cut on the original place with implanted titanium mesh for exposing titanium mesh. It can be clearly observed that a layer of new bone has been formed on titanium mesh surface. Experimental rabbits are killed. Ultrasonic knife can be used for removing titanium mesh from skull. The titanium mesh is placed in formalin liquid. The mesh is observed and compared subsequently. Different titanium mesh surface osteogenesis effects and osteogenesis thickness are measured. 


\section{Statistical Analysis}

Statistical analysis is implemented for measurement osteogenesis thickness data by statistical software SPSS 13.0. Calculated data is represented by $\mathrm{x} \pm \mathrm{s}$. $\mathrm{t}$ test is adopted for difference comparison among different groups. The analysis has significant difference if $\mathrm{P}<0.05$.

\section{Results}

\section{Specimen Osteogenesis Thickness Contrast}

Specimens are generally observed. Since the experiment is designed into six groups, namely: A. untreated titanium mesh and treated titanium mesh; B. untreated titanium mesh and untreated titanium mesh + cell patch; C. untreated titanium mesh and treated titanium mesh + cell patch; D. untreated titanium mesh + cell patch and processed titanium mesh + cell patch; E. untreated titanium mesh + cell patch and treated titanium mesh; F. treated titanium mesh and treated titanium mesh + cell patch. Since A experimental rabbit in the feeding process dies, five groups of experimental specimens are obtained only. However, since five groups contain A experimental rabbit specimen samples, data acquisition of the whole pattern is not affected, and photos of specimen in all groups are shown as follows (figure 4).

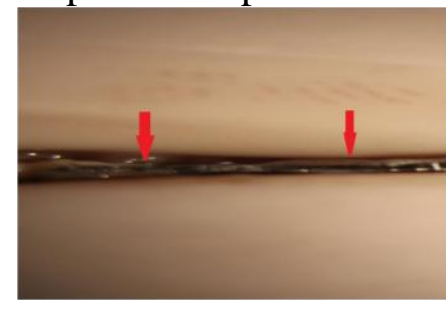

A

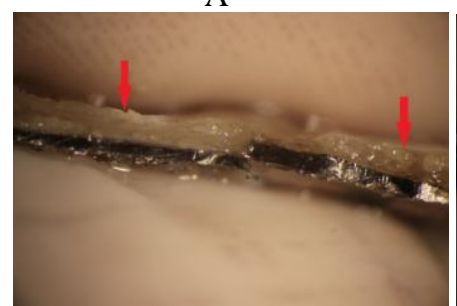

$\mathrm{D}$

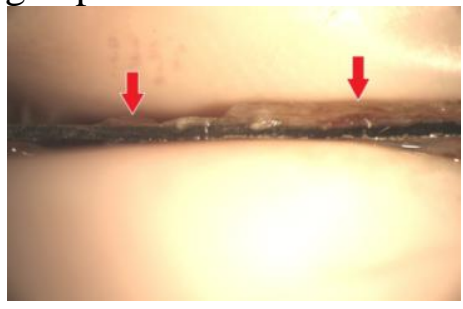

B

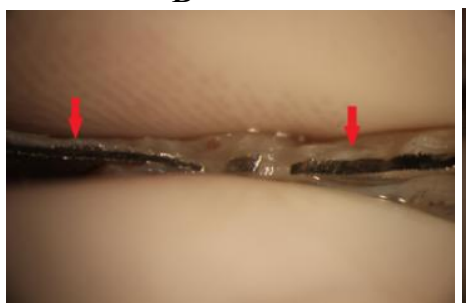

$\mathrm{E}$

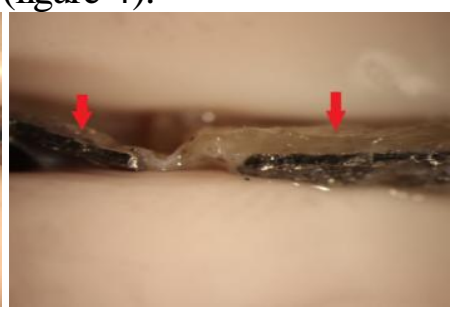

$\mathrm{C}$

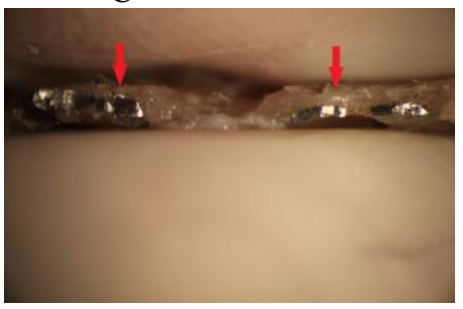

$\mathrm{F}$

Figure 4. Animal experimental results show. A.Left untreated titanium net, Right treated titanium net;B. Left untreated titaniumnet, Right untreated titanium mesh + cell membrane;C. Left untreated titanium net, Right treated titanium net + cell membrane;D. Left treated titanium net + cell membrane, Right untreated titaniummesh + cell membrane; E. Left treated titaniumnet, Right untreated titaniummesh + cell membrane;

F. Left treated titanium net, Right treated titanium mesh + cell membrane.

Table 1. Different surface structure titanium mesh and cell membrane combination osteogenesis measurements

\begin{tabular}{|c|c|c|}
\hline Titanium mesh & Cell patch & Osteogenesis thickness \\
\hline Without surface treatment & No & About $0.1 \mathrm{~mm}$ \\
\hline Without surface treatment & Yes & About $1.0 \mathrm{~mm}$ \\
\hline Surface treatment & No & About $0.3 \mathrm{~mm}$ \\
\hline Surface treatment & Yes & About $1.2 \mathrm{~mm}$ \\
\hline
\end{tabular}

\section{Specimen slice observation}

After general specimens are contrasted, titanium mesh on the specimen is removed, thin layer new bone can undergo pathology slice observation as shown in the following figure in order to 
further understand the osteogenesis effect on titanium mesh before and after titanium mesh surface SLA processing as well as before and after addition of bone marrow stem cell patch.

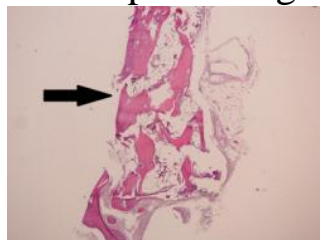

A

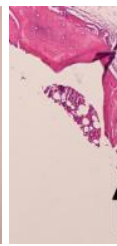

B

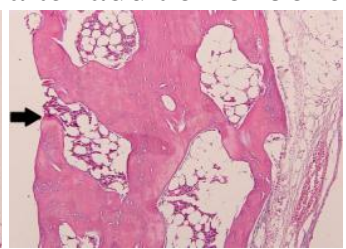

$\mathrm{D}$

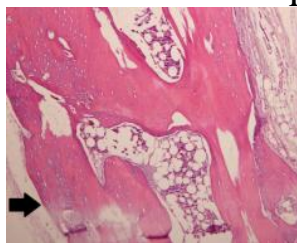

$\mathrm{C}$

Figure 5.New bone pathological on different titaniummesh surfacethe. A. Untreated titanium mesh surface osteogenesis;B. Treated titaniummesh surface osteogenesis; C. Untreated titanium mesh + cell membrane surface osteogenesis;D. Treated titanium mesh + cell membrane surface osteogenesis .

\section{Discussion}

\section{Reasons Why Bone Tissues also Can Be Produced in Some Parts of Titanium Mesh without Surface Processing under the Condition of No Cell Patch Function}

The result may be related to soft tissue covered by titanium mesh. Inner surface of some tissues may contain a few amount of periosteum tissues in soft tissues covered by titanium mesh. The periosteum tissue contains a lot of blood capillary. Mesenchymal stem cells are gathered together in the mode of osteogenesis in the membrane. When the cell masses grow to certain degree, the cells in the center of the mass are differentiated into osteogenesis cells. Then, osteogenesis cell secretes bone matrix as early bone. Early bone is not mature bone tissue. Mature bone tissue can be gradually formed through bone tissue reconstruction. In the process, after osteogenesis cells and osteoclasts act alternately and repeatedly, relatively complete and stable bone tissue structures are gradually produced. Slice observation is implemented on bone tissues produced on titanium mesh surface without SLA processing and not covered by cell patch in the experiment. It is discovered that the bone tissues are loose and disrupted. It also proves that the newly produced bone tissues can form stable structure through repeated reconstruction in the above theory. However, the bone tissue produced by titanium mesh surface without SLA processing and not covered by cell patch in the experiment is just located in the reconstruction stage. Therefore, the bone tissues are loose and disrupted. The titanium mesh surface after surface processing can produce more continuous bone structure. It is obvious that titaniummesh surface structure after SLA is more suitable for inside osteogenesis of periosteum. The reconstruction process of osteogenesis cells and osteoclasts to new bone tissues is also accelerated. Though there is no cell patch, new bone tissue still can be produced, and the process of producing new bone is more passive and slower.

\section{Cell Patch Function}

After titanium mesh surface is covered by the cell patch, no matter whether SLA processing is implemented on the titanium mesh surface, the inducing osteogenesis ability can be greatly improved. The point also can be comprehended well in fact. After cell patch is added, it is believed that a layer of periosteum is added in the tissue inner surface of soft tissue covered by the titanium mesh. In addition, the periosteal cells belong to cell layers which have been induced to the direction of osteogenesis cells. Therefore, when the cell activity is still high, the layers are directly covered on the titanium mesh surface, its ability for inducing and producing osteogenesis cells is also stronger. Therefore, when cell patch is covered on the titanium mesh 
for large area, the inducing osteogenesis effect is ideal. Therefore, so-called bionic bone structure is formed. Some clinical emergent problems are solved by the method. When patients are not willing to obtain bone for restoring jaw bone defect in other own parts, titanium mesh and cell patch technology can provide patients with ideal restoration plan.

After continuous bone tissue is formed on titanium mesh surface, osteogenesis membrane fiber tissue must be formed, material conditions are provided for facial muscle tendon adhesion.

\section{Research prospect}

Experiment result of inducing osteogenesis by applying bone marrow mesenchymal stem cell patch in titaniummesh surface: inducing osteogenesis is successful on one hand, it is difficult to cite it in clinical purpose on the other hand. Difficulties mainly include the follows: how to obtain bone marrow stem cell more conveniently and how to rapidly separate cell patch for cultivation in face of patients. If desired cells can be obtained from patient venous blood, treatment feeling of patients can be greatly improved. Next, how to obtain stem cell from patient blood and fat tissue is mainly studied in future research plan, and then osteogenesis stem cells can be effectively induced and cultivated. The treatment plan is further simplified, thereby providing clinical treatment with better treatment plan.

In summary, after titanium mesh with SLA treated surface is implanted into the bone defect part, the surface structure is changed, which is more suitable for inducing production of osteogenesis cells and formation of bone tissue. Homologous bone marrow stem cell patch is covered on the surface for obtaining excellent osteogenesis effect, and a feasible method can be provided for clinical treatment, which can be recommended.

\section{References}

[1] Bell RB. Computerplanning and intraoperative navigation in cranio-maxillofacial surgery.OralMaxillofacSurgClinNorth Am,2010,22(1):135-56.

[2] Ponader S, von Wilmowsky C, Widenmayer M, et al. (2010) In vivo performance of selective electron beam-melted Ti-6Al-4V structures. Journal of biomedical materials research Part A 92: 56-62.

[3] Bose S, RoyM, DasK, et al. Surface modification of titanium for load-bearing applications.J Mater Sci Mater Med.2009;20 Suppl 1:S19-24.

[4] Hannachi IE, Yamato M, Okano T. Cell sheet technology and cell patterning for biofabrication. Biofabrication, 2009, 1(2): 022002.

[5] Matsuda N, Shimizu T, Yamato M, et al. Tissue engineering based on cell sheet technology. Adv Mater, 2007, 19(20): 3089-3099.

[6] Van Den Dolder, Bancroft GN, Sikavitsas VI, et al. Flow perfusion culture of marrow stromal osteoblasts in titanium fiber mesh. Biomed Mater Res,2003,64A (2):235-241.

[7] Yang J, Yamato M, Kohno C, et al. Cell sheet engineering: recreating tissues without biodegradable scaff olds. Biomaterials, 2005, 26(33): 6415-6422.

[8] Becker W, Becker BE, Caffesse R, et al. A comparison of dematerialized freeze-dried bone and autologous bone to induce bone formation in human extraction sockets Periodontal, 1994;65:1128. 
[9] Han Bing, Fu Xiaobing. Research progress and clinical application prospect of mesenchymal stem cell. China Restoration and Reconstruction Surgery, 2006, 20(12): 1257-1261.

[10] Goldstein AS, Juarez TM, Helmke CD, et al. Effect of convection on osteoblastic cell growth and function in biodegradable polymer foam scaffolds.Biomaterials, 2001,22(11):1279-88. 